\title{
Papillary muscle infarction in relation to left ventricular infarct distribution and transmurality - assessment by delayed enhancement cardiac magnetic resonance imaging
}

\author{
Sean Wilson ${ }^{1 *}$, Fahmida Islam', Debbie W Chen ${ }^{1}$, Jason Chinitz', Parag Goyal', Kana Fujikura', Thanh Nguyen², \\ Yi Wang ${ }^{2}$, Robert A Levine ${ }^{3}$, Richard B Devereux', Jonathan W Weinsaft ${ }^{1,2}$
}

From 15th Annual SCMR Scientific Sessions

Orlando, FL, USA. 2-5 February 2012

\section{Summary}

This study used delayed enhancement CMR (DE-CMR) and invasive angiography to evaluate relationships between papillary muscle and left ventricular (LV) chamber wall infarction following ST segment elevation MI (STEMI). Results demonstrate that papillary muscle infarction (PMI) parallels infarct transmurality and contractile dysfunction within the adjacent LV wall.

\section{Background}

Papillary muscles and myocardium within the adjacent LV wall constitute two components of the mitral valve apparatus. Prior studies have demonstrated variable papillary arterial supply, and the relationship between PMI and overall LV infarct pattern is unknown. DECMR enables in-vivo study of infarct pattern within the LV - papillary muscle complex.

\section{Methods}

Patients with initial STEMI were enrolled in a prospective imaging registry. CMR (1.5T) was performed within 6 weeks ( $27 \pm 8$ days) post-STEMI. Cine-CMR (SSFP) was used to assess LV wall motion (17 segment model, 5 point per-segment score) DE-CMR (IR-GRE, acquired $10-30$ minutes post gadolinium $[0.2 \mathrm{mmol} / \mathrm{kg}]$ ) was used to assess infarct morphology: PMI was graded for location and extent (partial or complete, stratified by $>50 \%$ papillary hyperenhancement); LV infarction was

${ }^{1}$ Greenberg Cardiology Division/Departments of Medicine Weill Cornell Medical College, New York, NY, USA

Full list of author information is available at the end of the article quantified based on global size and regional transmurality (17 segment, 5 point per-segment score). Invasive coronary angiograms were read blinded to CMR.

\section{Results}

153 patients were studied, among whom $30 \%$ had PMI (74\% posteromedial/37\% anterolateral; $11 \%$ bilateral). Overall LV infarct size on DE-CMR was larger among patients with PMI ( $\mathrm{p}=0.01)$. PMI strongly related to LV infarct distribution (Table 1), with prevalence increased 3 -fold among patients with lateral wall, and over 1.5fold with inferior wall infarction on DE-CMR $(\mathrm{p} \leq 0.01)$. Angiography findings paralleled DE-CMR, with over a 2-fold increase in PMI with right coronary artery (RCA) or left circumflex (LCX) culprit vessel infarction $(\mathrm{p}<0.01)$. Among patients with RCA infarcts, PMI exclusively occurred $(100 \%)$ in the setting of right or codominant coronary anatomy and was associated with larger angiographic jeopardy score $(20.8 \pm 6.0$ vs. 15.8 $\pm 5.9, \mathrm{p}=0.007)$. In contrast, only one-third $(36 \%)$ with PMI and LCX infarcts were left or co-dominant, with similar jeopardy scores between patients with and without PMI (19.4 \pm 9.8 vs. $15.3 \pm 11.6, \mathrm{p}=0.45)$. Regarding extent, PMI was partial ( $\leq 50 \%$ hyperenhancement) in $76 \%$ of cases. PMI extent paralleled infarct transmurality in adjacent LV segments (Figure 1), with similar results when regional wall motion score was used as a surrogate for LV injury (all $\mathrm{p}<0.001$ ). Additionally, there was a stepwise increase in LV lateral wall infarct size $(\%$ myocardium) among patients with bilateral PMI (12.8 $\pm 4.2 \%)$ compared to those with isolated $(3.5 \pm 4.2 \%)$ or absent PMI $(0.8 \pm 2.0 \%)(\mathrm{p}<0.001$ for trend). 
Table 1 Infarct size and distribution

\begin{tabular}{|c|c|c|c|c|c|c|c|c|c|}
\hline & \multicolumn{3}{|c|}{ PMI } & \multicolumn{3}{|c|}{ Posteromedial PMI } & \multicolumn{3}{|c|}{ Anterolateral PMI } \\
\hline & $\begin{array}{c}\text { Present } \\
(n=46)\end{array}$ & $\begin{array}{l}\text { Absent } \\
(n=107)\end{array}$ & $\mathbf{P}$ & $\begin{array}{c}\text { Present } \\
(n=34)\end{array}$ & $\begin{array}{c}\text { Absent } \\
(n=107)\end{array}$ & $\mathbf{P}$ & $\begin{array}{l}\text { Present } \\
(n=17) \dagger\end{array}$ & $\begin{array}{l}\text { Absent } \\
(n=107)\end{array}$ & $\mathbf{P}$ \\
\hline \multicolumn{10}{|l|}{ INFARCT SIZE } \\
\hline \multicolumn{10}{|l|}{ DE-CMR } \\
\hline \% LV hyperenhancement & $19.5 \pm 11.4$ & $15.0 \pm 9.5$ & 0.01 & $19.2 \pm 11.4$ & $15.0 \pm 9.5$ & 0.03 & $23.5 \pm 14.3$ & $15.0 \pm 9.5$ & 0.002 \\
\hline \multicolumn{10}{|l|}{ Cardiovascular enzymes } \\
\hline Creatine phosphokinase & $2590 \pm 2344$ & $2164 \pm 1836$ & 0.25 & $2345 \pm 1751$ & $2164 \pm 1836$ & 0.63 & $3014 \pm 3282$ & $2164 \pm 1836$ & 0.34 \\
\hline Creatine phosphokinase-MB & $243 \pm 207$ & $199 \pm 189$ & 0.30 & $254 \pm 202$ & $199 \pm 188$ & 0.22 & $189 \pm 212$ & $199 \pm 188$ & 0.89 \\
\hline \multicolumn{10}{|l|}{ Duration of symptoms } \\
\hline Chest pain interval (hours) & $12.4 \pm 9.4$ & $10.5 \pm 8.4$ & 0.24 & $12.7 \pm 9.7$ & $10.5 \pm 8.4$ & 0.24 & $13.0 \pm 9.5$ & $10.5 \pm 8.4$ & 0.26 \\
\hline \multicolumn{10}{|l|}{ INFARCT DISTRIBUTION } \\
\hline \multicolumn{10}{|l|}{ DE-CMR } \\
\hline Anterior wall & $35 \%(16)$ & $70 \%(75)$ & $<0.001$ & $15 \%(5)$ & $70 \%(75)$ & $<0.001$ & $77 \%(13)$ & $70 \%(75)$ & 0.78 \\
\hline Lateral wall & $65 \%(30)$ & $22 \%(23)$ & $<0.001$ & $74 \%(25)$ & $22 \%(23)$ & $<0.001$ & $59 \%(10)$ & $22 \%(23)$ & 0.003 \\
\hline Inferior wall & $72 \%(33)$ & $45 \%(48)$ & 0.003 & $91 \%(31)$ & $45 \%(48)$ & $<0.001$ & $41 \%(7)$ & $45 \%(48)$ & 0.78 \\
\hline \multicolumn{10}{|l|}{$\begin{array}{l}\text { Invasive angiography (infarct } \\
\text { related artery) }\end{array}$} \\
\hline Left anterior descending & $28 \%(13)$ & $72 \%(77)$ & $<0.001$ & $3 \%(1)$ & $72 \%(77)$ & $<0.001$ & $71 \%(12)$ & $72 \%(77)$ & 1.00 \\
\hline Left circumflex artery & $24 \%(11)$ & $6 \%(6)$ & 0.001 & $32 \%(11)$ & $6 \%(6)$ & $<0.001$ & $24 \%(4)$ & $6 \%(6)$ & 0.03 \\
\hline Right coronary artery & $48 \%(22)$ & $22 \%(24)$ & 0.002 & $65 \%(22)$ & $22 \%(24)$ & $<0.001$ & $6 \%(1)$ & $22 \%(24)$ & 0.19 \\
\hline Anatomically dominant artery* & $57 \%(26)$ & $26 \%(28)$ & $<0.001$ & $77 \%(26)$ & $26 \%(28)$ & $<0.001$ & $6 \%(1)$ & $26 \%(28)$ & 0.12 \\
\hline
\end{tabular}

* Either left circumflex or right coronary artery.

† 5 subjects had concomitant posteromedial and anterolateral PMI.

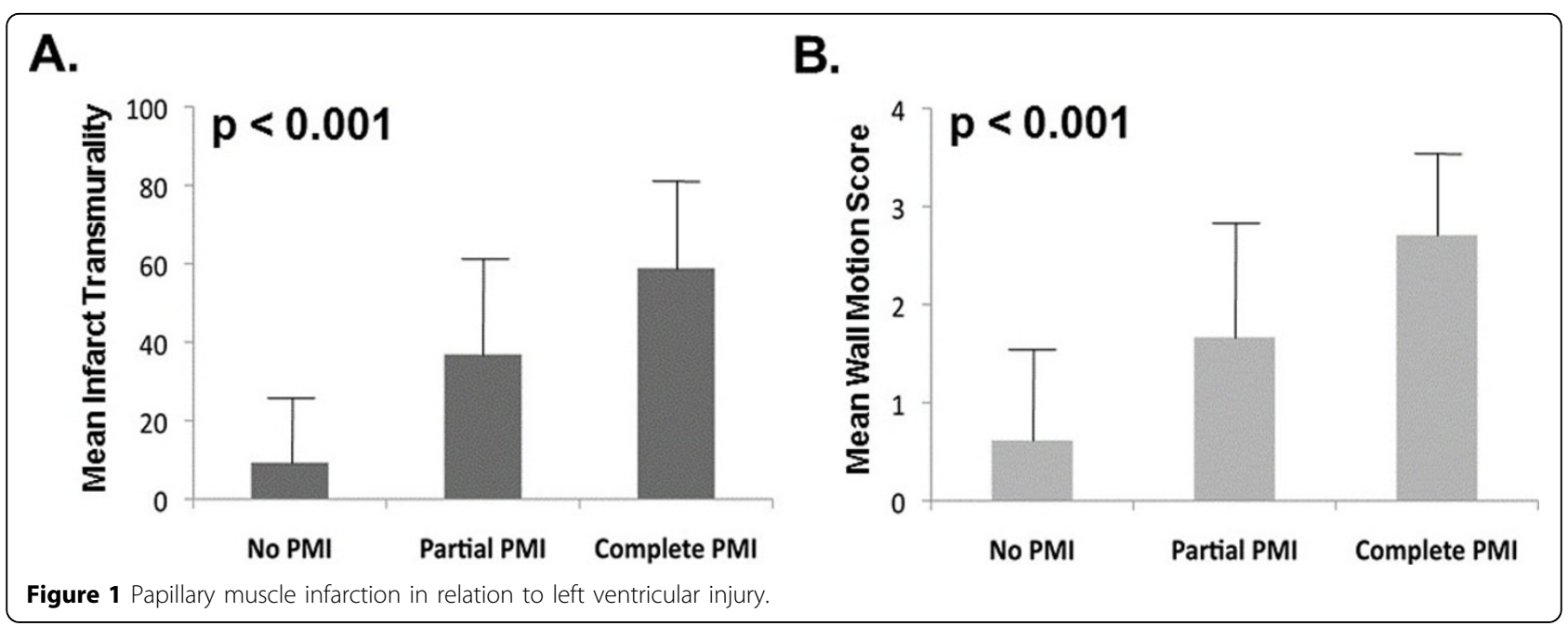

\section{Conclusions}

PMI is common following STEMI, with PMI extent paralleling infarct transmurality and contractile dysfunction within the adjacent LV wall. Current findings dispute the notion of papillary muscles as end-organ structures particularly susceptible to impaired perfusion, instead supporting the concept that papillary muscles and adjacent LV myocardium are similarly vulnerable to jeopardized arterial supply.

\section{Funding}

K23 HL102249-01, Lantheus Medical Imaging, Doris Duke Clinical Scientist Development Award (Jonathan W. Weinsaft, MD).

\section{Author details}

${ }^{1}$ Greenberg Cardiology Division/Departments of Medicine Weill Cornell Medical College, New York, NY, USA. ${ }^{2}$ Massachussetts General Hospital, Harvard Medical School, Boston, MA, USA. ${ }^{3}$ Radiology Weill Cornell Medical College, New York, NY, USA. 
doi:10.1186/1532-429X-14-S1-P36

Cite this article as: Wilson et al: Papillary muscle infarction in relation

to left ventricular infarct distribution and transmurality - assessment by

delayed enhancement cardiac magnetic resonance imaging. Journal of

Cardiovascular Magnetic Resonance 2012 14(Suppl 1):P36.

Submit your next manuscript to BioMed Central and take full advantage of:

- Convenient online submission

- Thorough peer review

- No space constraints or color figure charges

- Immediate publication on acceptance

- Inclusion in PubMed, CAS, Scopus and Google Scholar

- Research which is freely available for redistribution

Submit your manuscript at www.biomedcentral.com/submit
C Biomed Central 